\section{Precisión dimensional de una silicona de condensación: comparación del tiempo de almacenamiento y el método de medición}

\section{Dimensional accuracy of a condensation silicone: comparison of storage time and measurement method}

\section{Resumen}

Objetivo: El objetivo de este estudio fue medir el cambio dimensional de una silicona por condensación a través de diferentes instrumentos de medición (calibrador digital, estereomicroscopio y máquina de medición por coordenadas tridimensional) en cuatro diferentes tiempos. Métodos: En el estudio experimental in vitro se realizaron cuatro impresiones con silicona por condensación (Speedex) con un dispositivo específico con cuatro distancias, con un total de 16 mediciones llevadas a cabo con cada instrumento. Los tiempos predeterminados de medición fueron: inmediatamente después de la impresión (T0), después de 30 minutos (T1), 1 h (T2) y 7 días (T3) de la impresión. Las medias y desviaciones estándar de las mediciones se analizaron con prueba de varianza y la prueba de Tukey, con un nivel de significancia de 0,05. Resultados: La media (mm) para las muestras medidas con el calibrador digital $(\mathrm{T} 0=5,82 ; \mathrm{T} 1$ y T2 $=5,8 ; \mathrm{T} 3=5,78)$ y la máquina de medición por coordenadas tridimensional $(\mathrm{T} 0=5,75 ; \mathrm{T} 1=5,78$; $\mathrm{T} 2$ y $\mathrm{T} 3=5,77)$, presentaron diferencia estadísticamente significativa de la dimensión de los valores iniciales para los valores después de 7 días $(p=0,037 ; p=0,013$, respectivamente), la misma no se dio para el uso del estereomicroscopio (T0, T1 y T3= 5,83; y T2 $=5,81$ ), que presentó similitud estadística en todos los tiempos $(p=0,754)$. Conclusiones: El método de medición fue el factor relevante que influenció en los resultados de las dimensiones de las impresiones, los cuatro tiempos no fueron relevantes en los cambios dimensionales de la silicona de condensación analizada.

Palabras clave: Elastómeros de silicona; Materiales dentales; Precisión de la medición dimensional.

\begin{abstract}
Objective: This study aims to calculate the dimensional change of a condensation silicone through different measuring instruments (digital paquimeter, stereomicroscope and 3D coordinate measuring machine); in four separate times. Methods: In the experimental in vitro study four impressions of condensation silicone (Speedex) were performed with a specific device with four-time distances, resulting in sixteen measurements with each instrument. The pre-determinated times for measurement were: immediately after the impression (T0), after $30 \mathrm{~min}(\mathrm{~T} 1)$, after $1 \mathrm{~h} \mathrm{(T2)}$ and after 7 days (T3) of impression. The averages and standard deviations of measurements were submitted to analysis of variance and Tukey's test, with significance level of 0.05 . Results: The average (mm)
\end{abstract}

\section{Artículo Original}

Jaiane Bandoli Monteiro 1,a, Lisseth Patrícia Cláudio Contreras ${ }^{1, a}$, Thaiana Damasceno Cunha ${ }^{2, b}$, Luís Paulo Vasconcelos de Castro Leite $^{3, c}$, Jalon de Morais Vieira ${ }^{3, d}$, Ivone de Oliveira Salgado $2, \mathrm{e}$

${ }^{1}$ Universidade Estadual Paulista, São José dos Campos, Brasil.

${ }^{2}$ Universidade Federal de Juiz de Fora, Juiz de Fora, Brasil.

${ }^{3}$ Instituto Federal de Educação, Ciência e Tecnologia do Sudeste de Minas Gerais. Juiz de Fora, Brasil.

a Doctora en Materiales Odontológicos y Prótesis.

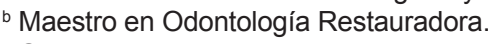

c Graduado en Ingeniería Mecatrónica.

d Doctor en Ingeniería Mecatrónica.

e Doctora en Odontología Restauradora.

\section{Correspondencia:}

Jaiane Bandoli Monteiro

Correo electrónico: jaiane.monteiro@ict.unesp.br Departamento de Materiales Odontológicos y Prótesis, Instituto de Ciencia y Tecnología - Universidad Estadual Paulista, Avenida Ing Francisco José Longo, número 777, Jardim São Dimas, São José dos Campos, São Paulo, Brasil. 12245-000. Teléfono: +55 32 988759734.

\section{Coautores:}

Patrícia Cláudio Contreras lisseth.contreras@ict.unesp.br

Thaiana Damasceno Cunha thaianacunha@yahoo.com.br Luís Paulo Vasconcelos de Castro Leite luis.engmecatronica@hotmail.com Jalon de Morais Vieira jalon.vieira@ifsudestemg.edu.br Ivone de Oliveira Salgado ivonne.deoliveira@ufjf.edu.br

Conflicto de intereses: Los autores declaran no tener conflictos de interés.

Fuente de financiamiento: Autofinanciado

Fecha de recepción: 09/06/18 Fecha de aceptación: 12/09/18 
for the samples measured with a digital paquimeter $(\mathrm{T} 0=5.82 ; \mathrm{T} 1$ y $\mathrm{T} 2=5.80 ; \mathrm{T} 3=5.78)$ and $3 \mathrm{D}$ coordinate measuring machine $(\mathrm{T} 0=5.75 ; \mathrm{T} 1=5.78$; $\mathrm{T} 2$ y $\mathrm{T} 3=5.77)$, there was a significant difference of dimension for the initial values in relation of values after 7 days $(\mathrm{p}=0.037 ; \mathrm{p}=0.013$, respectively), the same did not happen for the use of the stereomicroscope (T0, T1 y T3= 5.83; y T2= 5.81), that presented statistic similarity for all evaluated times $(p=0.754)$. Conclusions: The measurement method was the relevant factor that influenced the results of the dimensions of the impressions, the four times were not relevant in the dimensional changes of the silicon of condensation analyzed.

Keywords: Dental materials; Dimensional measurement accuracy; Silicone elastomers.

\section{Introducción}

El éxito de las restauraciones indirectas preparadas sobre un modelo de yeso depende de un minucioso diagnóstico, planificación y técnicas que incluyen etapas clínicas y de laboratorio, siendo que la transferencia de la situación clínica del paciente se basa en una adecuada toma de impresión ${ }^{1}$.

La toma de impresión es un paso de fundamental importancia en el tratamiento, debido a que la reproducción de las dimensiones reales de las estructuras dentales tiene como consecuencia restauraciones protésicas con ajuste marginal correcto ${ }^{2}$.

La calidad de las impresiones está influenciada por varios factores: técnica utilizada y su correcta ejecución, ubicación de la terminación de la preparación y propiedades de los materiales de impresión ${ }^{3}$. Entre las propiedades presentadas para el material de impresión, la exactitud en la reproducción detallada y la estabilidad dimensional (incluso cuando se almacena durante un largo periodo de tiempo) son claves para la elección del material por parte del profesional ${ }^{4}$.

Actualmente, los elastómeros son los materiales de impresión más comunes ${ }^{5}$. Entre estos, las siliconas por condensación son las más utilizadas por su eficacia y facilidad de acceso ${ }^{6}$, sin embargo, durante su polimerización, pierden subproductos volátiles como alcohol etílico, y en consecuencia, genera contracción del material y probablemente un cambio dimensional ${ }^{7,8}$. Uno de los principales factores asociados con este cambio dimensional es el período de tiempo entre la toma de impresión y el vaciado de la impresión para la obtención del modelo positivo ${ }^{9}$. Para evitar que los modelos de escayola obtenidos de la impresión de silicona por condensación presenten distorsión, es necesario que el vaciado se realice en el período de tiempo en el que el material todavía no haya sufrido contracción dimensional y se haya producido una recuperación de la deformación elástica, que permite el regreso a su tamaño original durante su remoción ${ }^{8}$.

Una vez que se trata de dimensiones muy pequeñas, la determinación de la precisión dimensional es un proceso crítico y está representada por el grado de reproducibilidad entre las mediciones ${ }^{10}$. Previamente estudios publicados han demostrado que las medidas lineales fueron utilizadas para investigar la exactitud de los modelos dentales ${ }^{11,12}$, sin embargo, los métodos introducidos son limitados por la falta de puntos de referencias estandarizadas.
Debido a la variedad de instrumentos de medición utilizados para evaluar los cambios dimensionales ocurridos en los materiales de impresión y considerando las limitaciones de esas medidas de precisión utilizadas anteriormente, el objetivo de este estudio fue medir los cambios dimensionales de impresiones de una silicona por condensación en el momento inmediato $(0 \mathrm{~s}$ después en la toma de impresión), 30 min, 1 a 7 días después del tiempo de polimerización y la obtención de la impresión, a través de instrumentos de medición específicos, como el calibrador digital, el estereomicroscopio y la máquina de medición tridimensional por coordenadas. Las hipótesis nulas probadas fueron: 1) que no habrá diferencia significativa en la precisión cuando cada tiempo se evalúe con los diferentes instrumentos y 2) no habrá diferencia significativa de la alteración dimensional en los tiempos determinados para la medición con cada uno de los instrumentos.

\section{Métodos}

La investigación fue realizada en el laboratorio del Núcleo de Mecánica del Instituto Federal de Educação, Ciência e Tecnologia Sudeste de Minas Gerais/Campus Juiz de Fora - Brasil (IFET). Para ello, se desarrolló un dispositivo metálico con la finalidad de ejecutar y estandarizar las impresiones.

La figura $1 \mathrm{~A}$ representa el dispositivo metálico de acero inoxidable utilizado en este estudio y sus respectivas dimensiones. Estuvo compuesto de dos partes: una parte formada por una moldura perforada, que contiene una varilla atornillada, dos orificios para la penetración de los pines guía de la otra parte y perforaciones que contribuyen a la salida y la reducción de las presiones internas del material de impresión durante la toma de impresión, creando retenciones alrededor (Figura 1B). La otra parte del dispositivo se compone de tres indentaciones preestablecidas, con dos topes laterales (que limitan verticalmente la inserción de la cubeta con el material, garantizando siempre la misma fuerza de compresión durante el moldeado y la estandarización de la misma cantidad de material en todos las impresiones) y un perno guía centralizado en cada batiente (garantizan que la cubeta penetre y se quita siempre en la misma posición, no permitiendo movimientos indeseables durante su remoción y/o distorsiones del molde) alineado con los orificios de inserción de los pines. La diferencia de altura entre los topes y las indentaciones fue de 4 $\mathrm{mm}$, espacio destinado al relleno con material de moldeo (Figura 1C). Los lugares medidos en las impresiones fueron copias negativas de las distancias entre las indentaciones de esta parte del dispositivo. 
Todas las impresiones fueron realizadas con silicona por condensación pesada y ligera Speedex Putty/Speedex Light Body (Coltene/Vigodent AS Industria y Comercio, Río de Janeiro, RJ, Brasil). Todos los materiales fueron manipulados de acuerdo con las recomendaciones del fabricante (proporción dos medidas de la base densa con seis medidas del catalizador, proporción 1:1 de la pasta ligera con el catalizador, tiempo de mezcla de 30 s y tiempo de presa final del material de 330 s). La manipulación de la pasta ligera con el catalizador fue hecha utilizando una espátula metálica de manipulación no 36 (Duflex,
SSWhite, Río de Janeiro, RJ, Brasil) y una placa de vidrio pulida de $15 \mathrm{~mm}$ (Prisma Instrumentos Odontológicos, Pirituba, SP, Brasil). El dispositivo con los pines guía fue insertado, adaptado en la cubeta y removido después del tiempo de polimerización del material.

Se realizaron cuatro impresiones para cada instrumento de medición $(\mathrm{N}=48)$. Después de cada toma de impresión, se utilizó únicamente un instrumento para realizar las medidas de la impresión. Se obtuvieron 16 medidas por cada instrumento en cada tiempo diferente. (Figura 2), medidos en cuatro tiempos: inmediatamente - $0 \mathrm{~s}$

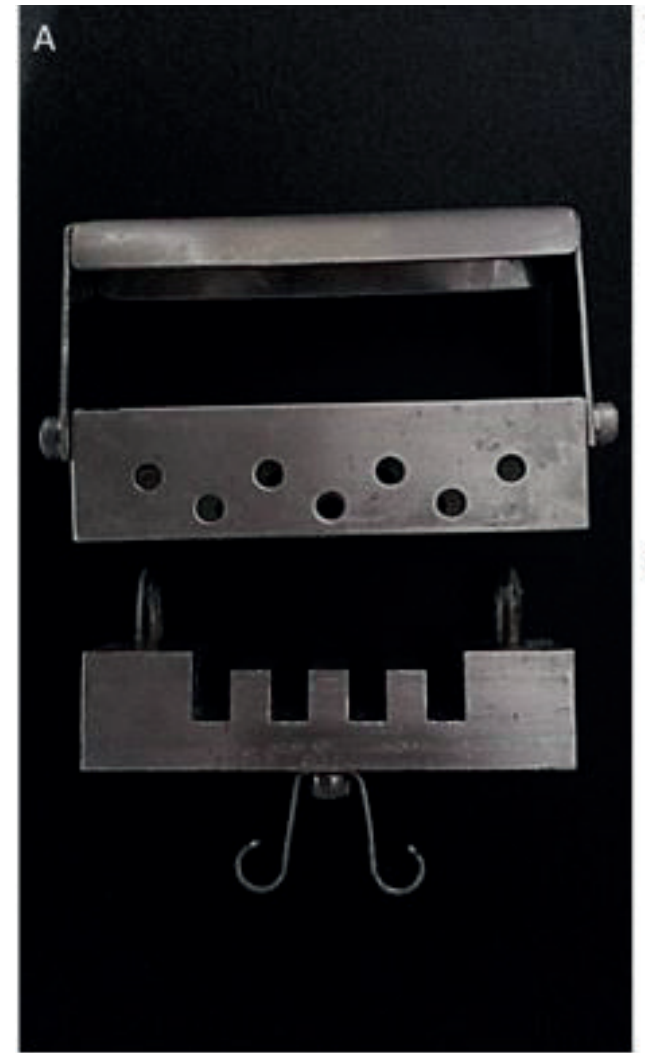

B
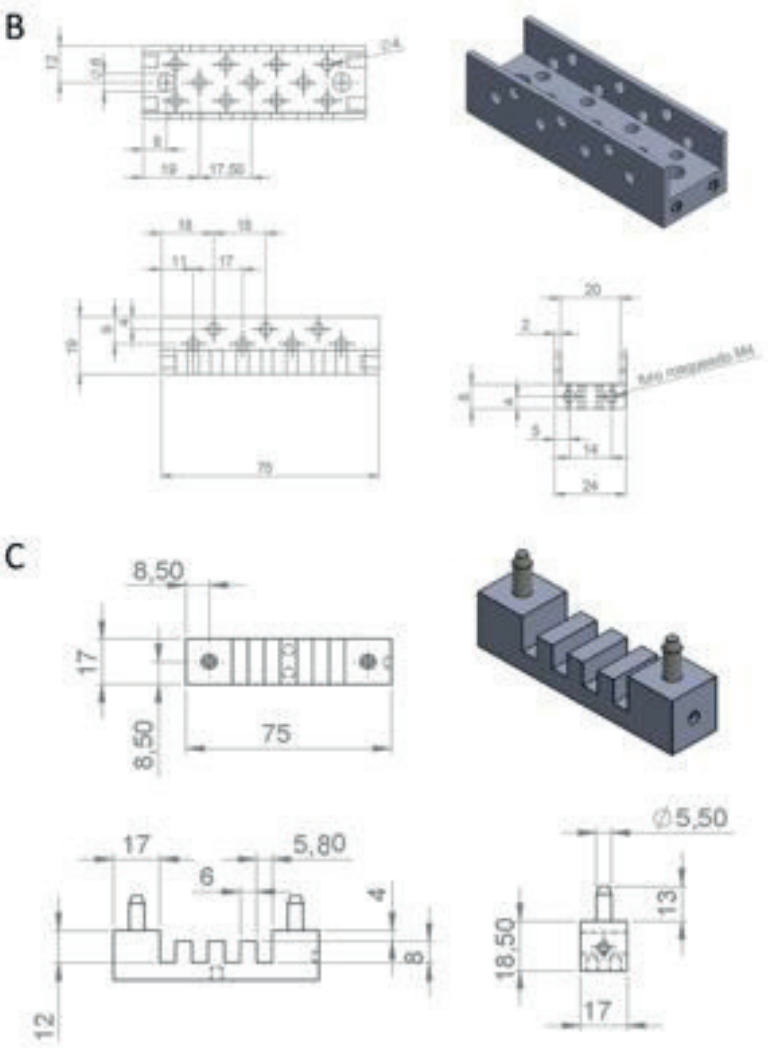

Figura 1. A. Dispositivo utilizado en la investigación con sus respectivas dimensiones (B y C)

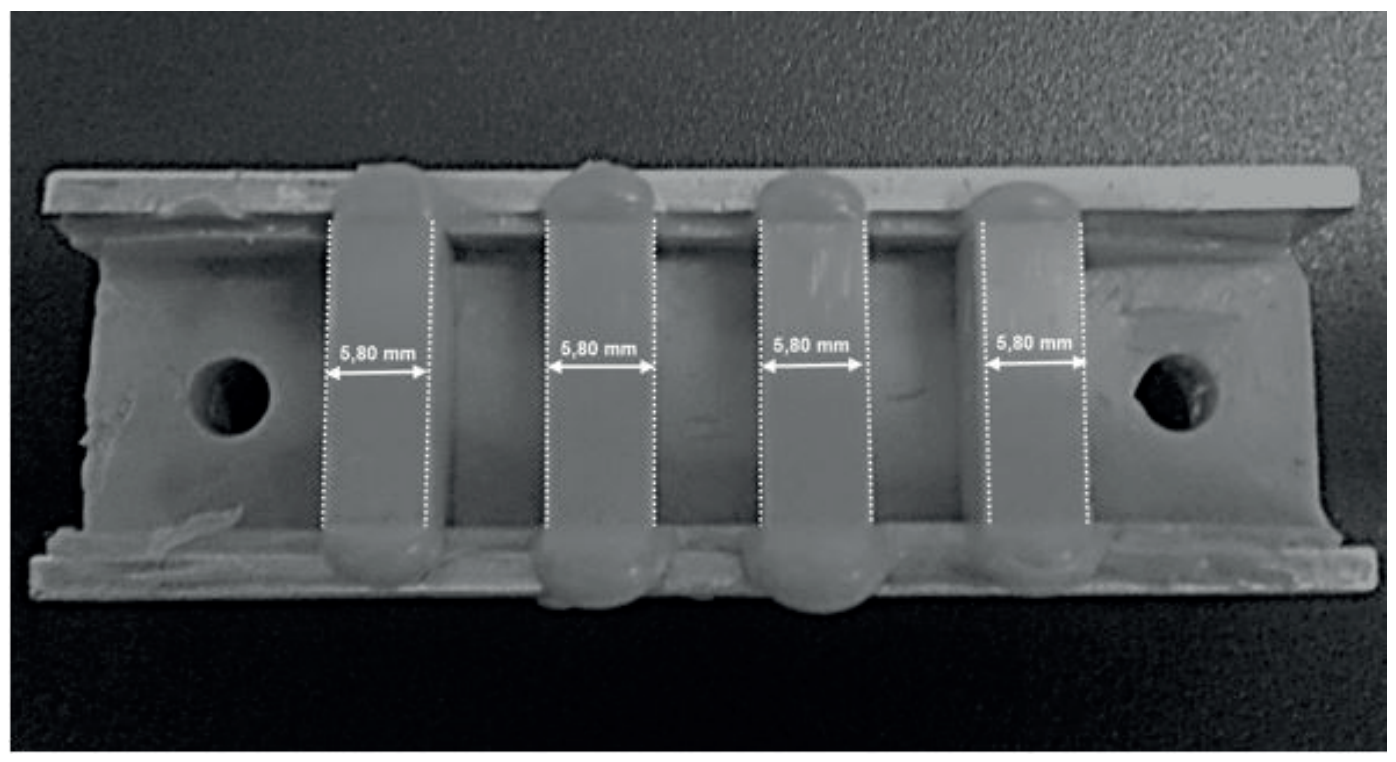

Figura 2. Representación esquemática de las cuatro medidas obtenidas en el molde 
(T1), 30 min (T2), 1 h (T3) y 7 días (T4) después del tiempo de polimerización y remoción del molde.

Todas las mediciones se realizaron en sala con temperatura ambiente de $(21 \pm 2){ }^{\circ} \mathrm{C}$ y humedad relativa del aire del $41 \%$. Durante todo el experimento, los moldes fueron debidamente almacenados en seco, a temperatura ambiente. Los instrumentos utilizados para realizar las mediciones fueron: calibrador digital Absolute (Mitutoyo, São Paulo, SP, Brasil), estereomicroscopio (Olympus modelo SZ61, iluminador Olympus LG-p52 y sistema de captura óptica Olympus SC 30, Tokio, Japón) con aumento de 10X y una máquina de medición tridimensional por coordenadas (Crysta-Plus M574, programa computacional MCOSMOS-1 versión 2.4, Mitutoyo, São Paulo, SP, Brasil, precisión dimensional de $10^{-3} \mathrm{~mm}$ ).

Después de cada impresión, las 16 mediciones se realizaron con cada respectivo instrumento. El perno fue desatornillado de la cubeta y la impresión fue colocada en el estereomicroscopio y se tomaron las micrografías de cada ángulo. Las mediciones realizadas con el calibrador digital fueron hechas en el lugar preestablecido con la punta móvil del calibrador posicionado de forma que la indentación se quedara con holgura entre la punta fija y la móvil. La parte móvil fue arrastrada levemente con el dedo pulgar hasta que se recostara suavemente en el material y luego la medida fue leída en la pantalla del calibrador digital. La impresión fue colocada en la máquina de medición tridimensional por coordenadas, la punta palpadora de la máquina tocó tres puntos para formar una recta en cada extremo de cada indentación rectangular y la medición se realizó con la distancia entre las rectas. A través de cada uno de los equipamientos, las mediciones se realizaron tres veces y la media arit- mética de esas medidas fue tomada como referencia y trazada en la tabla.

Para evaluar la relación entre los tiempos de medición con cada instrumento, los datos obtenidos fueron sometidos al modelo estadístico análisis de varianza 1 -factor, después de ser evaluadas las suposiciones del modelo de análisis de varianza. Los valores residuales, resultantes del ajuste del modelo adoptado, fueron examinados para evaluar la adecuación del modelo para válidas inferencias estadísticas y se determinó que los datos originales propiciaron un adecuado ajuste, porque los valores residuales se ajustan a una distribución normal de probabilidad, se verificó la uniformidad de los residuos (homocedasticidad) por medio de los valores residuo en relación a los valores ajustados. Los datos obtenidos y los resultados fueron sometidos al análisis de varianza (ANOVA 1-factor) y test de Tukey, con un nivel de significancia de 0,05 , utilizando el programa computacional Minitab 17 Statistical Software (Pensilvania, EUA).

\section{Resultados}

La tabla muestra los valores de la media (en $\mathrm{mm}$ ) de las 16 mediciones de la silicona por condensación, las desviaciones estándar y los intervalos de confianza correspondientes para todos los tiempos de almacenamiento para el calibrador digital, el estereomicroscopio y la máquina de medición tridimensional por coordenadas, respectivamente.

Para el caso de las muestras medias con el calibrador y la máquina de medición tridimensional por coordenadas, hubo diferencia significativa en los valores de medición inmediatamente después del moldeo (T0) $(5,82 \pm 0,03$ $\mathrm{mm}, 5,75 \pm 0,03 \mathrm{~mm}$; respectivamente) en relación a los valores dimensionales después de 7 días (T3) (5,78

Tabla. Media ( $\mathrm{mm}$ ), desviación estándar e intervalos de confianza de las mediciones realizadas con el calibrador digital, con el estereomicroscopio y con la máquina de medición tridimensional por coordenadas en los diferentes tiempos

\begin{tabular}{|c|c|c|c|c|}
\hline \multirow{2}{*}{ Instrumento } & \multirow{2}{*}{ Tiempo } & \multirow{2}{*}{ Media (mm) y D.E } & \multicolumn{2}{|c|}{ IC (95\%) } \\
\hline & & & Mínimo & Máximo \\
\hline \multirow{4}{*}{ Calibrador digital } & $\mathrm{T} 1$ (0 min) & $5,82 \pm 0,03^{A}$ & 5,80 & 5,84 \\
\hline & $\mathrm{T} 2$ (30 min) & $5,80 \pm 0,02^{\mathrm{A}, \mathrm{B}}$ & 5,79 & 5,82 \\
\hline & T3 (1 hora) & $5,80 \pm 0,04^{\mathrm{A}, \mathrm{B}}$ & 5,78 & 5,82 \\
\hline & T4 (7 días) & $5,78 \pm 0,04^{\mathrm{B}}$ & 5,76 & 5,80 \\
\hline \multirow{4}{*}{ Estereomicroscopio } & $\mathrm{T} 1$ (0 min) & $5,83 \pm 0,05^{\mathrm{A}}$ & 5,80 & 5,86 \\
\hline & $\mathrm{T} 2$ (30 min) & $5,83 \pm 0,06^{\mathrm{A}}$ & 5,80 & 5,86 \\
\hline & T3 (1 hora) & $5,81 \pm 0,07^{\mathrm{A}}$ & 5,78 & 5,84 \\
\hline & T4 (7 días) & $5,83 \pm 0,03^{\mathrm{A}}$ & 5,80 & 5,86 \\
\hline \multirow{4}{*}{$\begin{array}{l}\text { Máquina de medición tridimensional por } \\
\text { coordenadas }\end{array}$} & $\mathrm{T} 1$ (0 min) & $5,75 \pm 0,03^{\mathrm{A}}$ & 5,73 & 5,76 \\
\hline & $\mathrm{T} 2$ (30 min) & $5,78 \pm 0,03^{\mathrm{A}, \mathrm{B}}$ & 5,76 & 5,79 \\
\hline & T3 (1 hora) & $5,77 \pm 0,02^{\mathrm{A}, \mathrm{B}}$ & 5,75 & 5,78 \\
\hline & T4 (7 días) & $5,77 \pm 0,01^{\mathrm{B}}$ & 5,76 & 5,78 \\
\hline
\end{tabular}

D.E: Desviación estándar

Letras de superíndices diferentes significan diferencias estadísticamente significativas.

Las mismas letras superíndices indican igualdad estadística. 
$\pm 0,04 \mathrm{~mm}, 5,77 \pm 0,01 \mathrm{~mm} ; p=0,037$ y $p=0,013$ respectivamente) (Tabla). Lo mismo no ocurrió para el grupo probado con el estereomicroscopio, que presentó semejanza estadística desde el momento inicial de la medición hasta 7 días (Tabla) $(p=0,754)$, mas sólo con la observación en el estereomicroscopio hubo disminución dimensional de la silicona por condensación (T0 = 5,82 mm y T3 = 5,78 mm) (Figura 3).

\section{Discusión}

Mediante la toma de las impresiones se reproducen las estructuras dentales del paciente y se envían al laboratorio, este es un paso importante en el proceso de fabricación de restauraciones indirectas ${ }^{13}$. La calidad de las impresiones es fundamental para la adecuada adaptación de la restauración ${ }^{14}$, ya que errores en este proceso interfieran en todas las etapas posteriores ${ }^{9}$. Las impresiones pueden tener su calidad afectada por varios factores, como la técnica utilizada ${ }^{9}$, el material utilizado ${ }^{15}$ y el tipo de cubeta seleccionada para ejecutar la toma de impresión ${ }^{16}$. Para evitar los cambios ocurridos en la impresión en función de los factores arriba citados, en esta investigación se utilizó un dispositivo específico con características propias y estandarizadas que permitieron la confiabilidad de las impresiones, como descrito anteriormente por Monteiro et al. ${ }^{17}$.

La réplica precisa de las preparaciones en el diente y la posición del mismo en el arco dental requieren de mate- riales de impresión con alta estabilidad dimensional, ya que el vaciado de escayola inmediato no es posible cuando existe gran demanda de trabajos de restauraciones indirectas. En el caso de los elastómeros, la estabilidad dimensional depende directamente de su recuperación elástica, de la contracción, de la evaporación de componentes volátiles ${ }^{18,19}$ y de la contracción térmica de los mismos ${ }^{19}$. La estabilidad dimensional de los materiales de impresión puede verse afectada también por los productos y las técnicas de desinfección, así como por el tiempo de almacenamiento de estos, si no se siguen adecuadamente las instrucciones del fabricante ${ }^{8,20}$.

En el caso de los materiales elastoméricos utilizados en la clínica odontológica, podemos citar la silicona por condensación, que se caracteriza por presentar buena flexibilidad, recuperación elástica, en la reacción de polimerización se liberan subproductos volátiles, como el alcohol etílico y pueden ocasionar problemas con la precisión ${ }^{13}$, por lo que este estudio evaluó la alteración dimensional de una silicona por condensación después de diferentes tiempos de almacenamiento con diferentes instrumentos de medición. Los resultados mostraron que sólo con la utilización del estereomicroscopio no hubo diferencia significativa entre los tiempos de almacenamiento, rechazando parcialmente la primera hipótesis nula.

La liberación del alcohol etílico como subproducto genera contracción dimensional de la silicona polimeri-
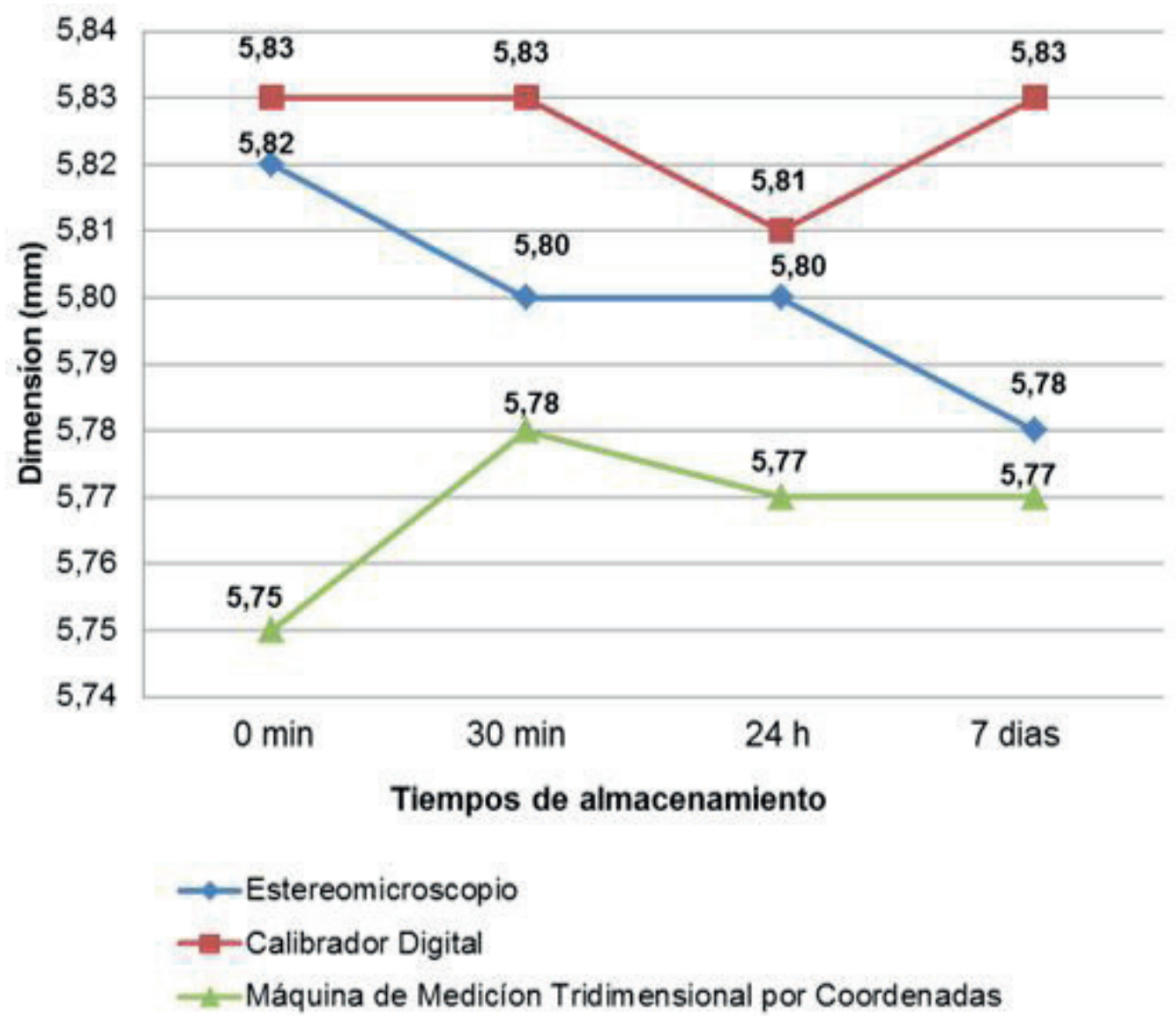

Figura 3. Representación gráfica de la media de la dimensión de acuerdo con el tiempo medido con los diferentes instrumentos 
zada por condensación, lo que no es deseable para los materiales de impresión. Papadogiannis et al. ${ }^{8}$ probaron que hubo alteración dimensional del material después del almacenamiento entre 30 min y 2 semanas y mostraron que ese tiempo debe ser limitado para las primeras $48 \mathrm{~h}$, pero para la silicona utilizada en ese estudio, la recomendación del vaciado del escayola puede ocurrir entre 30 y 7 días después de la realización de la toma de impresión ${ }^{13}$. De acuerdo con la figura 3, el material presentó expansión dimensional después de 30 min y $1 \mathrm{~h}$ de la toma de impresión, de acuerdo con las mediciones realizadas por la máquina de medición tridimensional por coordenadas, sin embargo, ocurrió contracción dimensional después de 30 min y $1 \mathrm{~h}$ de acuerdo con las mediciones realizadas por el estereomicroscopio. Con el calibrador digital, podemos notar que la dimensión después de 7 días permaneció idéntica a la inicial evidenciando que los instrumentos utilizados para las mediciones interfirieron en los resultados, fortaleciendo entonces el rechazo parcial de la primera hipótesis nula.

Aunque las modificaciones dimensionales en el material en los tiempos probados fueron significativas para las mediciones con los tres instrumentos, las medidas realizadas en el tiempo T0 (inmediatamente después del moldeado) en relación a las realizadas en el tiempo T3 (24 h) están dentro del límite máximo recomendado por la American Dental Association (ADA) para alteración dimensional de materiales de moldeo, que es de $0,5 \%$ de encogimiento en $24 \mathrm{~h}^{21}$. Por lo tanto, en este material se puede realizar el vaciado inmediatamente o después de 7 días de almacenamiento en seco a temperatura ambiente.

Se han reportado varias técnicas en la literatura para medir los cambios dimensionales de los materiales de impresión, incluyendo la utilización de fotografías realizadas por microscopios y cámaras fotográficas ${ }^{22}$ y diferentes instrumentos se pueden utilizar como compases de calibre digital ${ }^{23}$, micrómetros y paquímetros ${ }^{24,25}$. Todos estos instrumentos realizan mediciones bidimensionales. Además de estos, otros métodos para investigar la precisión de los modelos odontológicos por la medición de puntos de superficie con alta exactitud ya fueron estudiados, como la máquina de medición tridimensional por coordenadas ${ }^{13,26,27}$. El surgimiento de sistemas de medición tridimensional traía beneficios como el aumento de la exactitud y facilidad de operación, escaneado del tamaño y de la geometría del objeto ${ }^{28}$ a partir de puntos de sondeo medidos y transformados a través del procesamiento de seńal y el modelo de medición ${ }^{29}$. En este trabajo se utilizó una máquina de medición tridimensional por coordenadas, un calibrador digital y un estereomicroscopio. Los tres instrumentos utilizados en los diferentes tiempos de almacenamiento presentaron una diferencia estadísticamente significativa, por lo que la segunda hipótesis fue aceptada.

A pesar de la falta de estandarización entre los estudios, esta investigación siguió orientaciones de una revisión crítica de la literatura, que soporta las siguientes recomendaciones para controlar la estabilidad dimensional: las impresiones deben ser almacenadas a temperaturas entre $(21 \pm 2){ }^{\circ} \mathrm{C}$ y deben ser almacenados en un ambiente con humedad relativa inferior al 50\% durante todo el tiempo, hasta que se haya realizado el vaciado con escayola ${ }^{30}$.

Dentro de las limitaciones de este estudio, se puede concluir que: independientemente del instrumento usado para las medidas, de las impresiones con silicona hubo condensación dimensional sin cambios y los instrumentos para la medición fue el factor relevante, porque interfirieron en los resultados de la dimensión de las impresiones de silicona por condensación y para la medición con estereomicroscopio, el material presentó valores estadísticamente similares para todos los tiempos, lo que fue diferente para el calibrador digital y la máquina de medición tridimensional por coordenadas, que presentaron diferencia estadística entre los valores medidos inmediatamente después del moldeado y después de 7 días.

\section{Referencias bibliográficas}

1. Hamalian TA, Nasr E, Chidiac JJ. Impression materials in fixed prosthodontics: influence of choice on clinical procedure. J Prosthodont. 2011;20(2):153-60.

2. Chochlidakis KM, Papaspyridakos P, Geminiani A, Chen CJ, Feng IJ, Ercoli C. Digital versus conventional impressions for fixed prosthodontics: A systematic review and meta-analysis. J Prosthet Dent. 2016;116(2):18490 .

3. Balkenhol M, Haunschild S, Erbe C, Wöstmann B. Influence of prolonged setting time on permanent deformation of elastomeric impression materials. J Prosthet Dent. 2010;103(5):288-94.

4. Nassar U, Oko A, Adeeb S, El-Rich M, Flores-Mir C. An in vitro study on the dimensional stability of a vinyl polyether silicone impression material over a prolonged storage period. J Prosthet Dent. 2013;109:172-8.

5. Rubel BS. Impression materials: a comparative review of impression materials most commonly used in restorative dentistry. Dent Clin N Am. 2007;51:629-42.

6. Kalantari MH, Malekzadeh A, Emamic A. The effect of disinfection with sodium hypochlorite $0.5 \%$ on dimensional stability of condensation silicone impression materials of Speedex and Irasil. J Dent (Shiraz). 2014;15(3):98-103.

7. Hondrum SO. Changes in properties of nonaqueous elastomeric impression materials after storage of components. J Prosthet Dent. 2001;85(1):73-81.

8. Papadogiannis D, Lakes R, Palaghias G, Papadogiannis Y. Effect of storage time on the viscoelastic properties of elastomeric impression materials. J Prosthodont Res. 2012;56(1):11-8.

9. Gomez-Polo M, Celemin A, del Rio J, Sanchez A. Influence of technique and pouring time on dimensional stability of polyvinyl siloxane and polyether impressions. Int J Prosthodont. 2012;25(4):353-6.

10. Lee SJ, Betensky RA, Gianneschi GE, Gallucci GO. Accuracy of digital versus conventional implant impressions. Clin Oral Implants Res. 2015;26(6):715-9. 
11. Larson TD, Nielsen MA, Brackett WW. The accuracy of dual-arch impressions: A pilot study. J Prosthet Dent. 2002;87:625-7.

12. Brosky ME, Major RJ, DeLong R, Hodges JS. Evaluation of dental arch reproduction using three-dimensional optical digitization. J Prosthet Dent. 2003;90:434-40.

13. De Cesero L, Nogarett LM, Mota EG, Fracasso LM, Araujo CRP. Influence of elastic recovery time on the dimensional stability of polydimethylsiloxane (PDS). Polímeros. 2013;23(4):473-6.

14. Storey D, Coward TJ. The quality of impressions for crowns and bridges: an assessment of the work received at three commercial dental laboratories. Assessing qualities of impressions that may lead to occlusal discrepancies with indirect restorations. Eur J Prosthodont Restor Dent. 2014;22(1):11-8.

15. Thota KK, Jasthi S, Ravuri R, Tella S. A comparative evaluation of the dimensional stability of three different elastomeric impression materials after autoclaving - an invitro study. J Clin Diagn Res. 2014;8(10):ZC48-50.

16. Kumar V, Aeran H. Evaluation of effect of tray space on the accuracy of condensation silicone, addition silicone and polyether impression materials: an in vitro study. J Indian Prosthodont Soc. 2012;12(3):154-60.

17. Monteiro JB, Garcia CR, Salgado IO, Chaves-Netto HDM. Evaluación de la dimensión lineal de siliconas por condensación a través de la medición de coordenadas en tres dimensiones. Acta Odontol Venezolana. 2015; 53(3). [Consultado el 17 de mayo 2018]. Accesible en: https:/www.actaodontologica.com/ediciones/2015/3/

18. Franco EB, da Cunha LF, Benetti AR. Effect of storage period on the accuracy of elastomeric impressions. J Appl Oral Sci. 2007;15(3):195-8.

19. Pande NA, Parkhedkar RD. An evaluation of dimensional accuracy of one-step and two-step impression technique using addition silicone impression material: An in vitro study. J Indian Prosthodont Soc. 2013;13(3):254-9.

20. Martin N, Martin MV, Jedynakiewicz NM. The dimensional stability of dental impression materials following immersion in disinfecting solutions. Dent Mater. 2007;23(6):760-8.
21. American Dental Association. Council of Dental Materials and Devices. Specification no 19 for non-aqueous, elastomeric dental impression material. J Amer Dent Assoc. 1977 ; 94(4):733-41.

22. Sinobad T, Obradović-Djuricić K, Nikolić Z, Dodić S, Lazić V, Sinobad V, et al. The effect of disinfectants on dimensional stability of addition and condensation silicone impressions. Vojnosanit Pregl. 2014;71(3):251-8.

23. Chandran DT, Jagger DC, Jagger RG, Barbour ME. Two- and three-dimensional accuracy of dental impression materials: effects of storage time and moisture contamination. Biomed Mater Eng. 2010;20(5):243-9.

24. Leifert MF, Leifert MM, Efstratiadis SS, Cangialosi TJ. Comparison of space analysis evaluations with digital models and plaster dental casts. Am J Orthod Dentofacial Orthop. 2009;136(1):16.e1-4.

25. Pereira JR, Murata KY, Valle AL, Ghizoni JS, Shiratori FK. Linear dimensional changes in plaster die models using different elastomeric materials. Braz Oral Res. 2010;24(3):336-41.

26. DeLong R, Heinzen M, Hodges JS, Ko CC, Douglas WH. Accuracy of a system for creating 3D computer models of dental arches. J Dent Res. 2003;82:438-42.

27. Nakhaei M, Madani AS, Moraditalab A, Haghi HR. Three-dimensional accuracy of different impression techniques for dental implants. Dent Res J (Isfahan). 2015;12(5):431-7.

28. Lee SJ, Betensky RA, Gianneschi GE, Gallucci GO. Accuracy of digital versus conventional implant impressions. Clin Oral Implants Res. 2015;26(6):715-9.

29. Calvo R, D’Amato R, Gómez E, Domingo R. Integration of error compensation of coordinate measuring machines into feature measurement: Part I - Model Development. Sensors (Basel). 2016;16(10):1610.

30. Gonçalves FS, Popoff DA, Castro CD, Silva GC, Magalhães CS, Moreira AN. Dimensional stability of elastomeric impression materials: a critical review of the literature. Eur J Prosthodont Restor Dent. 2011;19(4):163-6. 